\title{
Evaluating foods and diets from a multi-dimensional perspective: nutrition, health and environment
}

\section{Abstract}

Introduction

The study of the environmental impact of dietary patterns in relation to their nutrition quality and health impact is of recent development and great interest for both nutrition and environmental scientists. Preliminary research has been conducted on the potential application of nutrition and health indexes as reference units (i.e. functional units) in the life cycle assessment of foods. Although proposed methods to include nutrition and health aspects exist, more research is needed to increase the scientific quality and societal usefulness of such assessments.

\section{Materials and Methods}

SLF Healthy Diets is a 20-month project where 64 food items from the FFQ used in the population study "Västerbotten Intervention Programme" (VIP) will be characterized for their nutrition density and matched to the carbon footprint from life cycle assessment studies. Several nutrition density scores will be analyzed, among which the NRF9.3 score, a dietary-dependent NQI score, and a new nutrient index tailored for the Swedish population. Hazard ratios for total mortality will be estimated for 100.000 participants to the VIP study, and associations with reported intake of food products, nutrient density and environmental performance described.

\section{Results and Discussion}

Multiple results are expected from the project, among which a synthesis of the combined nutritional and environmental performance of the analyzed foods according to different methods, and hence the identification of the best nutritional index to apply in environmental studies. Additionally, the assessment in the VIP cohort of the associations between reported intake of food products and observed health outcomes will evaluate the ability of the suggested nutrition scores to predict the total mortality in the studied population.

The present project will allow for more robust quantification and communication of food products' sustainability performance. Specifically, the project will: develop clear advice on which nutrition scores can best be used in LCA food studies; evaluate pros and cons of combined environmental, nutritional, and health metrics; validate nutrition and health metrics ability to predict health outcomes within a Swedish population-based cohort; investigate and propose how combined environmental, nutritional and health metrics can be implemented and used by food chain stakeholders.

\section{Conflict of Interest}

There is no conflict of interest 